\title{
Real-time interactive ptychography from electron event representation data
}

Philipp Pelz ${ }^{1}$, Peter Ercius ${ }^{2}$, Colin Ophus $^{3}$, Ian Johnson $^{2}$ and Mary Scott ${ }^{1}$

${ }^{1}$ UC Berkeley, Berkeley, California, United States, ${ }^{2}$ Lawrence Berkeley National Laboratory, United States, ${ }^{3}$ Lawrence Berkeley National Laboratory, California, United States

The arrival of direct electron detectors (DED) with high frame-rates in the field of scanning transmission electron microscopy has enabled many experimental techniques that require the collection of a full diffraction pattern at each scan position, a field which is subsumed under the name 4D-STEM.

DED frame rates approaching $100 \mathrm{kHz}$ place stringent requirements on data transmission rates and computing infrastructure. Current commercial DEDs allow the user to make compromises in pixel bit depth, detector binning or windowing to reduce the per-fame file size and allow higher frame rates.

This change in detector specifications requires decisions to be made before data acquisition that may reduce or lose information that could have been advantageous during data analysis. The 4D Camera, a DED with $87 \mathrm{kHz}$ frame-rate developed at Lawrence Berkeley National Laboratory, reduces the raw data to a linear-index encoded electron event representation (EER) [1].

In this talk we show with experimental data from the 4D camera that linear-index encoded EER [2,3] and its direct use in 4D-STEM phase-contrast imaging methods enables real-time, interactive phase-contrast from large-area 4D-STEM datasets.

We detail the computational complexity advantages of the EER and the necessary computational steps to achieve real-time interactive ptychography using commonly available hardware accelerators.

Real-time reconstruction with direct phase-contrast imaging methods allows fast data exploration during the experiment and generation of an accurate initial guess for probe aberrations in iterative reconstruction methods.

Fig. 1 shows the current timings achieved for a 4Dcamera dataset with 512 x 512 scan positions. The centering and cropping step can be performed directly on the electron events, allowing a 40-100x speedup over the dense format for sparse electron events. The data is then binned to $<32 \times 32$ detector pixels and densified to prepare for the single-sideband reconstruction [4]. The single-sideband reconstruction consists of an element-wise multiplication and 4D- >2D reduction step and can be parallelized efficiently on the GPU. By keeping the data on the GPU all the time, we achieve reconstruction times < 1s, allowing for interactive tuning of aberrations and performing interactive virtual defocus-scans. The code is complemented with a Jupyter graphical user interface, such that all computation can be done remotely. [5]

\section{References}

[1] stempy: Toolkit for processing 4D STEM image data on HPC

[2] Guo, H. et al. Electron-event representation data enable efficient cryoEM file storage with full preservation of spatial and temporal resolution. IUCrJ 7, (2020).

[3] Datta, A. et al. A data reduction and compression description for high throughput time-resolved electron microscopy. Nature Communications 12, 664 (2021). 


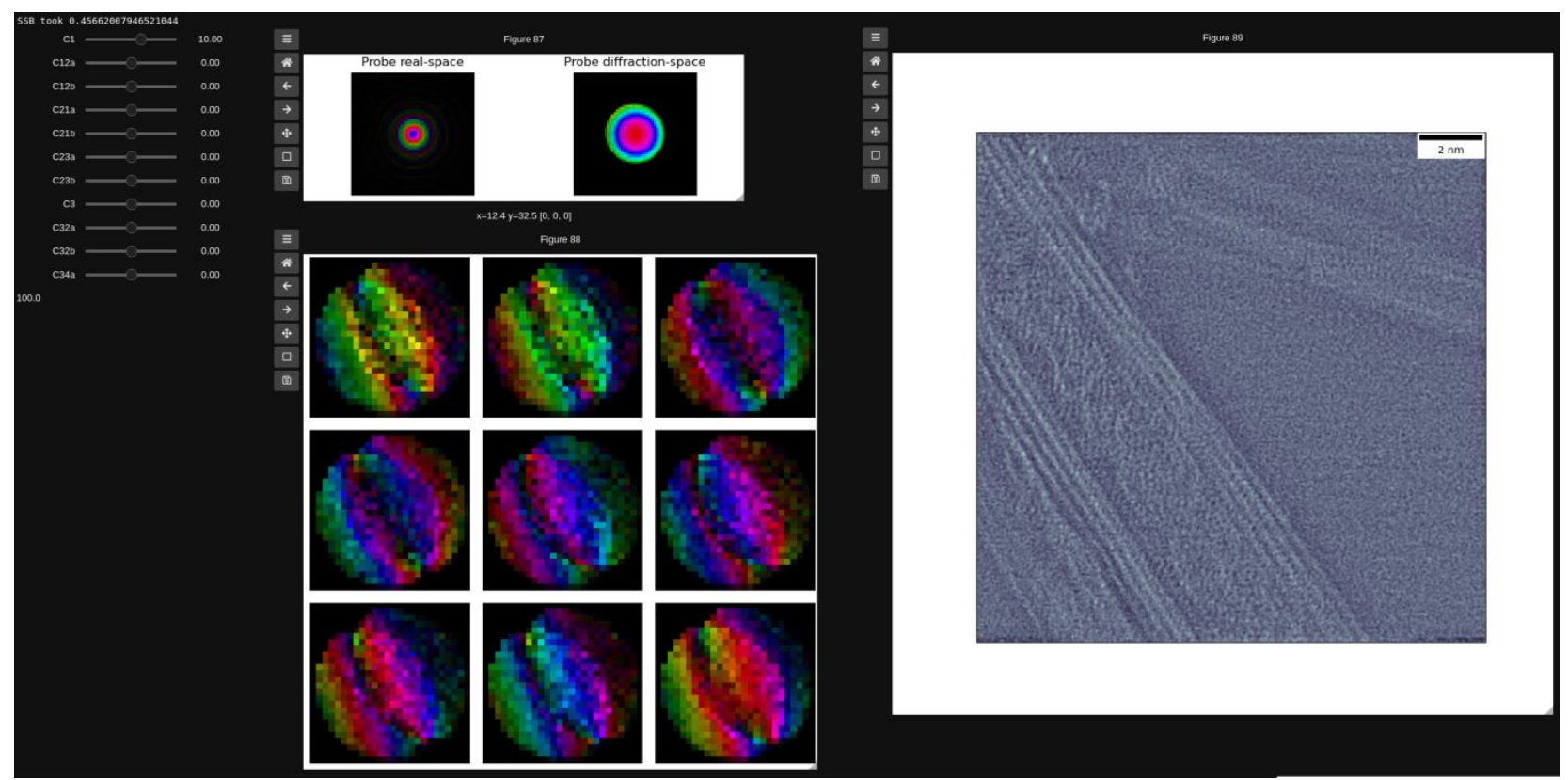

Figure 1. Fig 1: Graphical user-interface for real-time ptychography running in a jupyter-notebook. Left panel: interactive aberration tuning. Middle top panel: display of the current real- and diffraction-space probe aberrations. Middle bottom panel: Panel of the 9 most intensive double-overlap regions for aberration tuning feedback. Right panel: single-sideband reconstruction with the current aberrations.

\section{Current timing for $512 \times 512$ scan:}

\begin{tabular}{|l|l|}
\hline Operation & time \\
\hline Load from RAM into analysis PC GPU RAM & $<1 \mathrm{~s}$ \\
\hline Centering \& cropping & $24 \mathrm{~ms}$ \\
\hline Sparse to dense 4d cube & $17 \mathrm{~ms}$ \\
\hline SSB ptychography & $17 \mathrm{~ms}$ \\
\hline
\end{tabular}

Total: $<1 \mathrm{~s}$

Figure 2. Fig 2: Processing times for interactive ptychography. The sparse linear-index EER format allows small file size of < 2GB for a 512x521 4DSTEM-scan. Loading into GPU memory takes less than $1 \mathrm{~s}$, and the subsequent processing steps can be achieved with custom GPU kernels in under 100ms. This allows live updates of aberration parameters.

[4] Rodenburg, J. M., McCallum, B. C. \& Nellist, P. D. Experimental tests on double-resolution coherent imaging via STEM. Ultramicroscopy 48, 304-314 (1993).

[5] Work at the Molecular Foundry was supported by the Office of Science, Office of Basic Energy Sciences, of the U.S. Department of Energy under Contract No. DE-AC02-05CH11231. CO acknowledges additional support from the U.S. Department of Energy Early Career Research Program. M. S. and P. P. acknowledge support from the STROBE NSF Science and Technology Center on RealTime Functional Imaging. 